\title{
Electrophysiological Recording of Potassium Currents in Hippocampal Neurons
}

\author{
Yuxiang $\mathrm{Wu}^{1, \mathrm{a}}$, Minfang Huang ${ }^{1, \mathrm{~b}}$, Tao Song ${ }^{1, \mathrm{c}}$ and Guodong $\mathrm{Xu}^{1,2, *}$ \\ ${ }^{1}$ School of Physical Education, Jianghan University, Wuhan 430056, China \\ ${ }^{2}$ School of Health Sciences, Wuhan Institute of Physical Education, Wuhan 430079, China

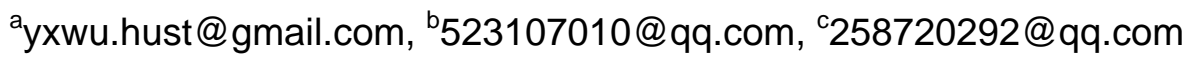 \\ *Author for correspondence: gdxbox@21cn.com
}

Keywords: potassium current, electrophysiology, neuron.

\begin{abstract}
Potassium channels are one of the most complex and wide-distributed channels in neurons, which have concentrated more and more attention, because it is an important signal pathway in the neuronal apoptosis and pathogenesis of Alzheimer's disease and neuron apoptosis. In this study whole-cell electrophysiological recording techniques were used to identify potassium currents in cultured rat hippocampal neurons (P7 to P14). The membrane potential was held at $-65 \mathrm{mV}$, before the activation of outward potassium currents, the membrane potential was pre-hyperpolarized to $-110 \mathrm{mV}$, and then depolarized to $+70 \mathrm{mV}$ in $10 \mathrm{mV}$ steps. We found that a kind of outward delayed potassium current was voltage dependent, and it could be significantly inhibited by $50 \mathrm{mM}$ tetraethyl ammonium (TEA).
\end{abstract}

\section{Introduction}

Ion channels in the cell membrane are the only way ions transporting into and out of the cell, which plays an important role in the regulation of cellular function [1, 2]. Potassium channels are one special kind of membrane proteins widely existed in cells. With the development of cell patch clamp and single channel recording technique, genetic recombination technology, such as channel protein separation and purification of the vigorous the application of biochemical technology, many channel protein molecular structure has been gradually clear [3]. Potassium channels are known to be most numerous types of ion channels, most widely distributed ion channels and one of the most complex types of ion channels, which have many functions, such as participating in the regulation of heart rate, smooth muscle contraction and secretion of the hormone, and the regulation mechanism is complex.

In the nervous system hippocampal neurons are composed of several types of potassium channels, which together form the potassium currents in the brain [4]. According to the characteristics of these currents, they can be roughly divided into the delayed rectifier and transient outward potassium currents [5, 6]. Transient outward potassium current was originally recorded and named by Conner and Stevens in Aplysia neurons [7]. So far, in mammalian cells found at least five expressions of potassium ion channel current subunits: $\mathrm{Kv} 1.4, \mathrm{Kv} 3.4, \mathrm{Kv} 4.1, \mathrm{Kv} 4.2$ and $\mathrm{Kv} 4.3$, which have the important significance of pathology, physiology and pharmacology. Intracellular potassium efflux is an important feature of apoptosis [2, 8, 9]. In many pathological conditions, reduced concentration of intracellular potassium can cause neuronal apoptosis, potassium channel blockers or extracellular potassium can block neuronal apoptosis [10-12]. However, there is no good answer about what kind of potassium ion channels are involved in neuronal apoptosis and how to regulate the potassium channels in the apoptotic process [13].

\section{Materials and methods}

Hippocampus from new born Sprague-Dawley rats within 24 hours were dissected and grown in culture dish at the density of 10000 cells/ $\mathrm{cm}^{2}$ as previously described [14, 15]. Electrophysiological recordings were carried out at room temperature using a double-patch clamp amplifier (Axon-700B, 
CA, USA). Patch pipettes were filled with internal solution containing (mM): $\mathrm{KCl} 140, \mathrm{MgCl}_{2} 2.5$, EGTA 10.0, HEPES 10.0 and ATP 4. The external solution in which neurons were bathed containing (mM): $\mathrm{NaCl}$ 125.0, $\mathrm{KCl} 3.0, \mathrm{CaCl}_{2}$ 2.5, $\mathrm{MgSO}_{4} 2.4, \mathrm{NaHCO}_{3} 26.0$ and $\mathrm{KH}_{2} \mathrm{PO}_{4}$ 1.5. Both solutions were adjusted with the osmotic pressure to $320 \mathrm{mOsm} / \mathrm{L}$ and the $\mathrm{PH}$ valve to 7.4. The resistance of the recording pipettes was in the range of 2-6 M . Statistical analyses of electrophysiological recording data were performed using Clampfit and SigmaPlot 11.0.

\section{Electrophysiological recording of neurons}

After seven days culture, the hippocampal neurons were matured for experiments. The neurons suitable for recording were $20-40 \mu \mathrm{m}$ in diameter with smooth show and in good contrast (Shown in Figure 1). The membrane potential was held at $-65 \mathrm{mV}$ and the low-pass Bessel filter set at 2,000 $\mathrm{Hz}$. When a giga-seal is carefully formed, the pipette capacitance, liquid junction potential to the bath and fast capacity transients are compensated. After the membrane of the neuron is ruptured, the slow capacity transients should be compensated and the whole cell recording is achieved. Moving average method is used and the cutoff frequency of Butterworth low-pass filter is $10 \mathrm{~Hz}$.

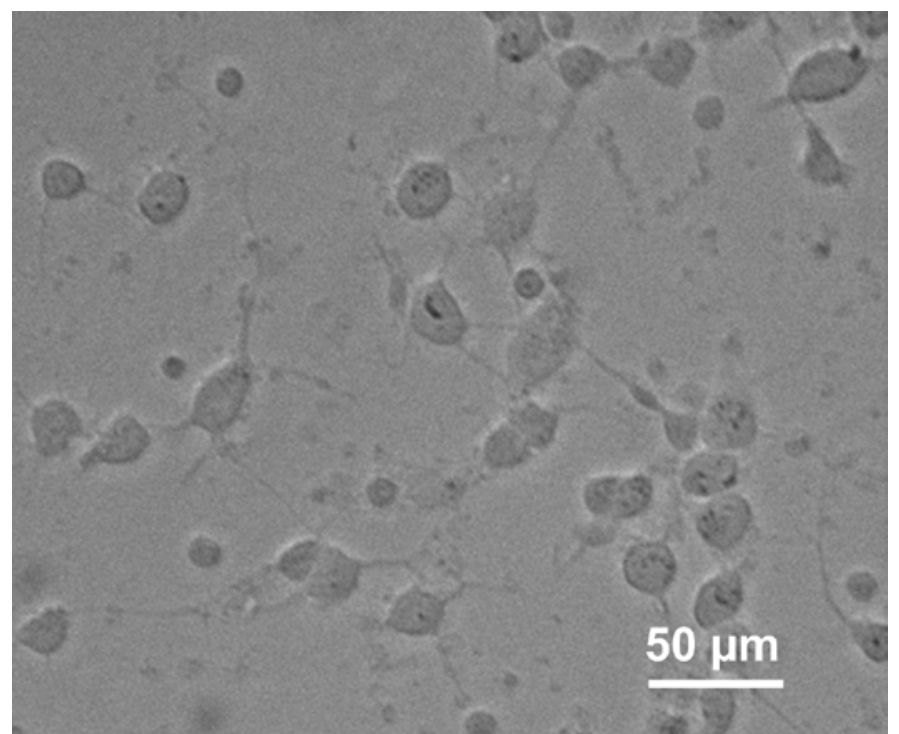

Fig. 1 Cultured hippocampal neurons for electrophysiological recording.

\section{Delayed outward potassium currents in hippocampal neurons}

As shown in Figure2, $500 \mathrm{~ms}$ hyperpolarized step to $-110 \mathrm{mV}$ was set to inhibit interference currents, and then a series of $2000 \mathrm{~ms}$ step by step was set from -70 to $+70 \mathrm{mV}$ in $10 \mathrm{mV}$ increments. Finally, the membrane potential restore to the resting potential. Through the activation process, the outward potassium currents were elicited and measured. The activation time of potassium channel was less than $5 \mathrm{~ms}$, and the currents were voltage-dependent and sensitive to TEA. 5 minutes after adding 50 $\mathrm{mM}$ TEA into the incubation dish, the outward currents were significantly inhibited for more than $65 \%$. Fast activation and fast inactivation is its typical characteristics in neuronal cell. When the membrane is depolarized about $-65 \mathrm{mV}$, potassium current is activated rapidly. After activation of about $1 \mathrm{~ms}$, the inactivation gate starts to work. The fast potassium current component completely inactivated at the membrane potential of $-45 \mathrm{mV}$, Thus during the current activation to - $45 \mathrm{mV}$ mainly fast potassium current components were recorded. Between $-45 \mathrm{mV}$ and $+70 \mathrm{mV}$ membrane potential, slow potassium current components were recorded. The fast and slow potassium current components are of great significance for regulating the action potential and adjusting conduction of hippocampal neurons signaling. 


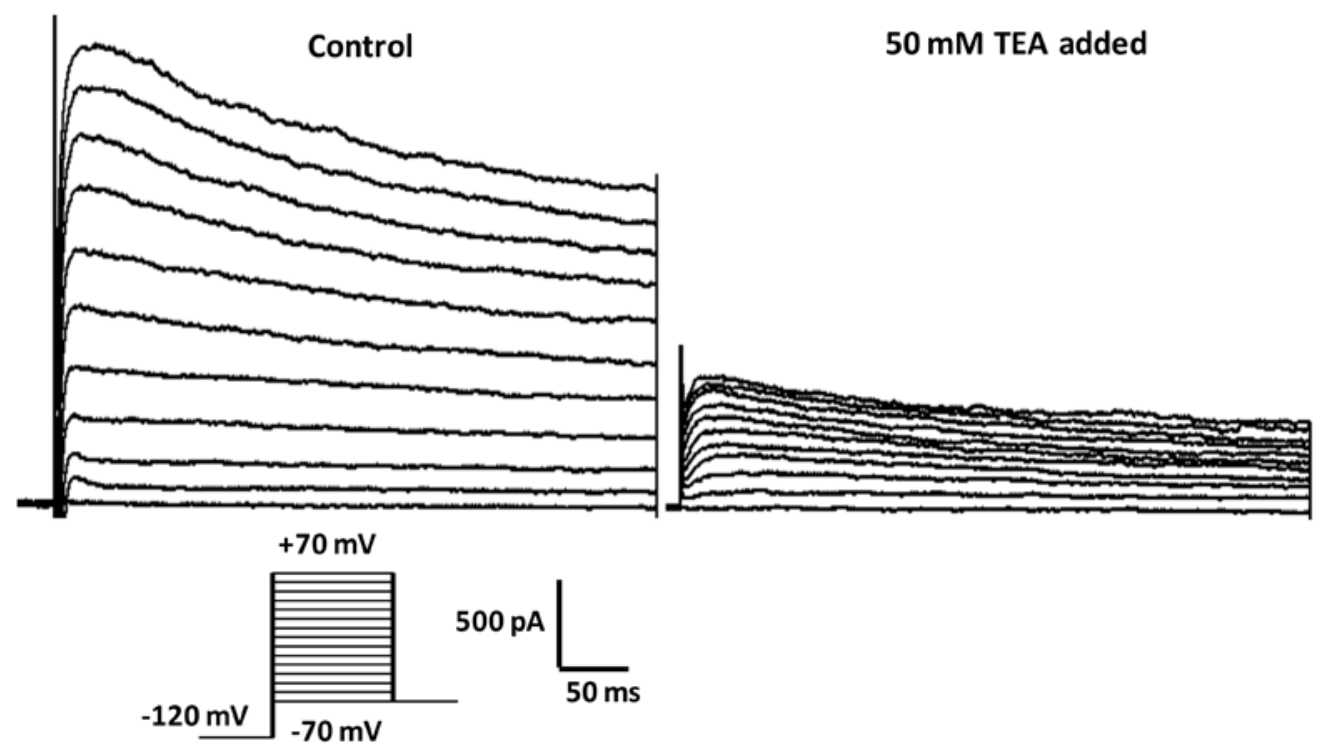

Fig. 2 Membrane currents in response to depolarizing voltage steps and $50 \mathrm{mM}$ TEA.

According to Ohm's law, voltage is equal to current multiplied by resistance. Thus the slope of the $\mathrm{I} / \mathrm{V}$ curve is the conductance (g), which is a measure of how many ions the channel can pass during a certain time. Current-voltage (I-V) curve of potassium ion channels revealed that the reversal membrane potential is closed to the potassium equilibrium potential, and the voltage dependent delayed outward potassium is sensitive to TEA as shown in Figure 3. To calculate the channel currents, electrophysiological data were $80 \%$ of compensated and filtered with a frequency of $2 \mathrm{kHz}$. Seal resistances were typically over $1 \mathrm{G} \Omega$, and the neurons input resistances were between 100 and 4 $\mathrm{M} \Omega$ after establishing the whole-cell recording. Correction for linear leak currents was done by subtracting a scaled current elicited by a $10 \mathrm{mV}$ hyperpolarizing step (at a holding potential of - 110 $\mathrm{mV}$ as described). Leak currents were defined by recording the currents in response to an inverted voltage command waveform.

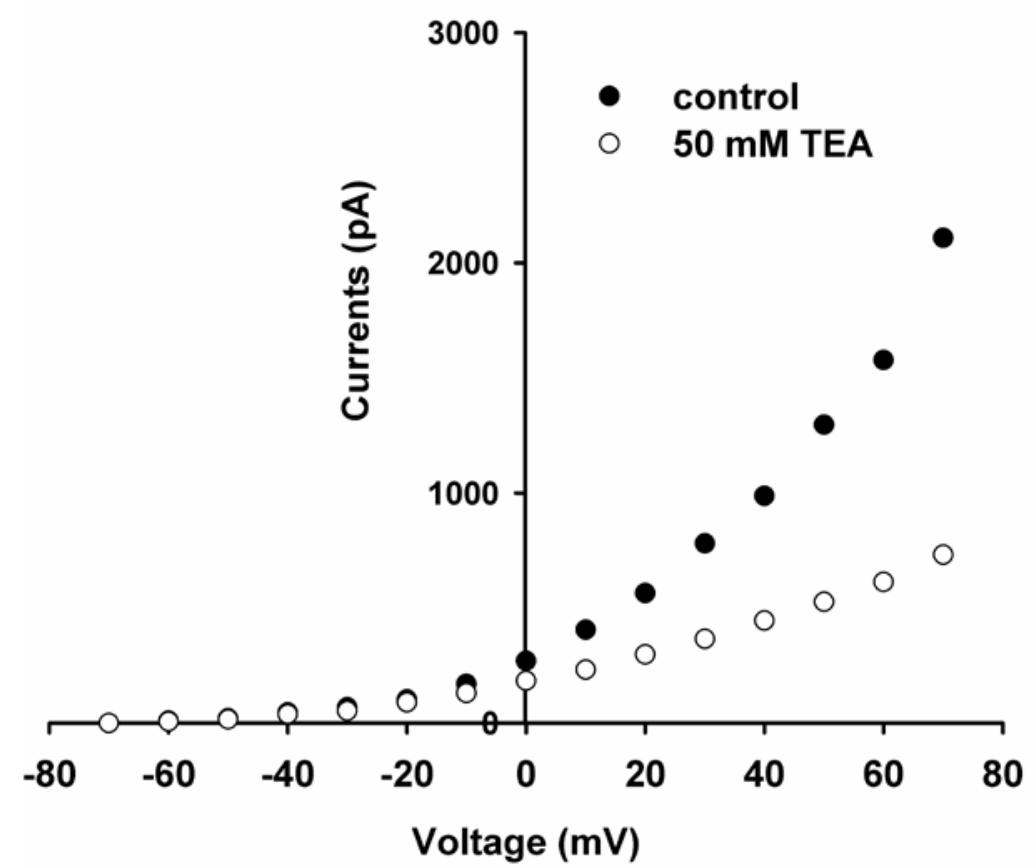

Fig. 3 Current-voltage (I-V) curve of potassium ion channels in hippocampal neurons. 


\section{Conclusions}

At present, Potassium channels are now known to be the most complex and widely distributed ion channels, due to its significant importance in the pathology, physiology and pharmacology, potassium currents are paid more and more attention, especially in the neuronal apoptosis and pathogenesis of Alzheimer's disease. The molecular level study makes us understand more about this channel. However, there are still some problems need to be further explored, such as function, gene mutation and disease subunit inconsistency. Due to complexity of potassium channel and its diversity, there are some limitations on potassium structure and functions. Therefore, to explore the early diagnosis and drugs for diseases, combined multidisciplinary research and advanced techniques have very important theoretical and practical significance. In this study, electrophysiological and pharmacological methods were combined to investigate the potassium channel characteristics of hippocampal neurons. We focus on the explanation of mechanism of potassium channels and demonstrated $50 \mathrm{mM}$ TEA potently inhibited outward delayed potassium currents of hippocampal neurons. Besides, both fast component and slow component of potassium currents are sensitive to the TEA. These findings suggest that the inhibition of outward delayed potassium currents in neurons could be probably accomplished through voltage-gated channels. As is known to all that intracellular potassium efflux and reduced concentration of intracellular potassium are very important features of cell apoptosis, our finding may provide some potential ways to the treatment of cell apoptosis. Besides, the new finding about the regulatory mechanism of potassium current in hippocampal neurons will have important significance for deepening our understanding of synaptic transmission, central nervous system, signal processing and integration, learning and memory and a series of physiological and pathological processes.

\section{Acknowledgements}

The study was supported by grants from the Educational Commission of Hubei Province of China (B2013154).

\section{References}

[1] L. Y. Jan, Y. N. Jan, J. Physiol. 590(2012) 2591-2599.

[2] J. Xu, P. Wang, Y. Li, P. Natl. Acad. Sci. USA. 101(2003)3112-3117.

[3] C. Gajewskia, A. Dagcanb, B. Roux, P. Natl. Acad. Sci. USA. 108(2011)3240-3245.

[4] Y. Jiang, A. Lee, J. Chen, Nature. 417(2002) 515-522.

[5] D. A. Doyle, J. M. Cabral, R. A. Pfuetzner, Science. 280(1998) 69-77.

[6] K. Yoshihiro, J. B. Timothy, Y. N. Jan, Nature. 362(1993) 127 - 133.

[7] J. A. Connor, C. F. Stevens, J. Physiol. 213 (1971)1-19.

[8] S. Grissmer, A. N. Nguyen, J. Aiyar, Mol. Pharmacol. 45(1994) 1227-1234.

[9] K. M. Tan, V. A. Lennon, C. J. Klein, Neurology. 70(2008) 1883-1890.

[10] R. Chen, A. Robinson, D. Gordon, Biophys. J. 101(2011) 2652-2660.

[11] P. Sergouniotis, A. E. Davidson, D. S. Mackay, Am. J. Hum. Genet. 89(2011) 183-190.

[12] T. Hoshi, Y. Tian, R. Xu, P. Natl. Acad. Sci. USA. 110(2013) 4822-4827.

[13] W. A. Catterall, S. Cestèle1, V. Yarov-Yarovoy, Toxicon. 49(2007)124-141.

[14] G. J. Brewer, J. Neurosci. Meth. 71(1997) 143-155.

[15] J. Ray, D. A. Peterson, M. Schinstine, P. Natl. Acad. Sci. USA. 90(1993) 3602-3606. 